\title{
Desenvolvimento de Novos Inibidores da Enzima Diidroorotato Desidrogenase
}

\section{(DHODH) de Leishmania sp.}

\author{
Development of New Inhibitors of the Dihydroorotate Dehydrogenase Enzyme (DHODH) from
}

Leishmania sp.

\author{
Desarrollo de Nuevos Inhibidores de la Enzima Dihidroorotato Deshidrogenasa (DHODH) de \\ Leishmania sp.
}

Recebido: 09/11/2021 | Revisado: 16/11/2021 | Aceito: 23/11/2021 | Publicado: 04/12/2021

\author{
Jorddy Neves da Cruz \\ ORCID: https://orcid.org/0000-0003-0529-3714 \\ Universidade da Amazônia, Brasil \\ E-mail: jorddynevescruz@gmail.com \\ Lucas Barbosa da Silva \\ ORCID: https://orcid.org/0000-0002-3921-5311 \\ Universidade da Amazônia, Brasil \\ E-mail: lucasbsilva261999@gmail.com \\ João Soares de Sousa Filho \\ ORCID: https://orcid.org/0000-0001-8320-7645 \\ Universidade da Amazônia, Brasil \\ E-mail: jhone.ss.filho@gmail.com \\ Joyce Karen Lima Vale \\ ORCID: https://orcid.org/0000-0001-9509-7009 \\ Universidade da Amazônia, Brasil \\ E-mail: joy.farmc@gmail.com
}

\begin{abstract}
Resumo
A leishmaniose é uma doença negligenciada sendo considerada uma das maiores endemias do mundo. Os parasitas causadores da doença desenvolveram resistência contra os medicamentos utilizados na farmacoterapia da doença. Devido a isso, novos compostos precisam ser planejados para tratar com eficácia essa parasitose. Sendo assim, neste paper utilizamos abordagens de modelagem molecular para planejar novos inibidores da enzima Diidroorotato desidrogenase (DHODH), alvo molecular contra leishimaniose. Inicialmente utilizamos modelagem molecular por homologia para construir a enzima DHODH, posteriormente o Metronidazol e Benzonidazol foram utilizados como arcabouços moleculares para desenvolvimento dos novos compostos. Em seguida, o método de docking molecular foi aplicado para avaliar o modo de interação dos compostos com o sítio de ligação da enzima. O modelo enzimático obteve $96 \%$ de seus resíduos em regiões favoráveis durante seu processo de validação. Nas simulações de docking, os compostos foram capazes de interagir favoravelmente com o local de ligação e exibiram interaç̃̃es com resíduos da proteína.
\end{abstract}

Palavras-chave: Leishmaniose; DHODH; Novos inibidores; Nitroderivados.

\begin{abstract}
Leishmaniasis is a neglected disease and is considered one of the greatest endemic diseases in the world. The diseasecausing parasites developed resistance against the drugs used in the disease's pharmacotherapy. Because of this, new compounds need to be devised to effectively treat this parasitosis. Therefore, in this paper we use molecular modeling approaches to design new inhibitors of the enzyme Dihydroorotate dehydrogenase (DHODH), a molecular target against leishmaniasis. Initially we used molecular modeling by homology to build the DHODH enzyme, later Metronidazole and Benznidazole were used as molecular frameworks for the development of new compounds. Then, the molecular docking method was applied to evaluate the interaction mode of the compounds with the binding site of the enzyme. The enzymatic model obtained $96 \%$ of its residues in favorable regions during its validation process. In the docking simulations, the compounds were able to interact favorably with the binding site and exhibit interactions with protein residues.
\end{abstract}

Keywords: Leishmaniasis; DHODH; New inhibitors; Nitro derivatives.

\section{Resumen}

La leishmaniasis es una enfermedad desatendida y se considera una de las mayores enfermedades endémicas del mundo. Los parásitos causantes de la enfermedad desarrollaron resistencia contra los fármacos utilizados en la 
farmacoterapia de la enfermedad. Debido a esto, es necesario diseñar nuevos compuestos para tratar eficazmente esta parasitosis. Por lo tanto, en este artículo utilizamos enfoques de modelado molecular para diseñar nuevos inhibidores de la enzima Dihidroorotato deshidrogenasa (DHODH), un objetivo molecular contra la leishmaniasis. Inicialmente usamos el modelado molecular por homología para construir la enzima DHODH, luego se usaron metronidazol y benznidazol como marcos moleculares para el desarrollo de nuevos compuestos. Luego, se aplicó el método de acoplamiento molecular para evaluar el modo de interacción de los compuestos con el sitio de unión de la enzima. El modelo enzimático obtuvo el $96 \%$ de sus residuos en regiones favorables durante su proceso de validación. En las simulaciones de acoplamiento, los compuestos pudieron interactuar favorablemente con el sitio de unión y exhibir interacciones con residuos de proteínas.

Palabras clave: Leishmaniasis; DHODH; Nuevos inhibidores; Derivados nitro.

\section{Introdução}

A leishmaniose é considerada uma das maiores endemias mundiais atingindo cerca de 98 países, dentre os quais 72 são classificados como países em desenvolvimento. Segundo a Organização Mundial da Saúde (OMS) são registrados anualmente cerca de 700.000 a 1 milhão de novos casos da doença (WHO, 2020). Essa doença é causa por protozoários da família Trypanosomatidae que fazem parte do gênero Leishmania e sua transmissão pode ocorrer pela picadura de insetos do gênero Phlebotomus ou Lutzomyia (Thakur et al., 2020).

Vinte e uma espécies de Leishmania são registradas como causadoras de leishmaniose em humanos, sendo que a doença pode ser categorizada de acordo com os locais do corpo que são afetados, como leishmaniose tegumentar, que ataca a pele e mucosas e leishmaniose visceral, que ataca órgãos internos (Akhoundi et al., 2016).

A leishmaniose tegumentar apresenta como sintomas clássicos o surgimento de um pequeno nódulo no local da picada do inseto que pode se transformar numa pequena ferida. Essas pequenas feridas possuem características que as distingue de outras feridas comuns da pele, como: sua consistência é endurecida, suas bordas são elevadas e seu fundo é avermelhada (Limeira et al., 2019).

A leishmaniose visceral possui como sintomas febre intermitente que se estendem por várias semanas e atingem temperaturas de aproximadamente $38^{\circ} \mathrm{C}$, outros sintomas são: inchaço e dores na região abdominal devido ao aumento de tamanho do fígado e baço, perda de pesa, nódulos linfáticos inchados, problemas respiratórios e diarreia (Arenas et al., 2017).

Na Amazonia Legal (Pará, Maranhão, Rondônia, Tocantins e Amazonas), Nordeste (Bahia), Sudeste (Minas Gerais e São Paulo), Centro-Oeste (Goiás) e Sul (Paraná) observou-se que a leishmaniose é causada principalmente pelo parasita Leishmania amazonensis (Ministério da Saúde, 2017).

O diagnóstico da doença pode ser realizado de diferentes maneiras, por exemplo, exame de lâminas a partir de material biológico obtido em biopsia ou raspagens dérmicas, crescimento de Leishmania em cultura, PCR para detecção do parasita e testes sorológicos para detecção de anticorpos contra Leishmania (CDC's Division of Parasitic Diseases and Malaria, 2014).

Para o tratamento da leishmaniose a farmacoterapia é limitada, basicamente há dois medicamentos para seu tratamento, sendo os Antimoniais pentavalentes (SbV) e Anfotericina B. Atualmente não há vacina disponível para o tratamento da leishmaniose, embora potenciais vacinas estejam em fases de teste pré-clínicos e clínicos (No, 2016).

Os medicamentos Antimoniais usados na farmacoterapia contra a leishmaniose são altamente tóxicos e há também o surgimento de espécies de Leishmania sp. que possuem resistência as drogas usadas (Mandlik \& Singh, 2016). Devido isso, é necessário o desenvolvimento de novas drogas com menor toxicidade e que para as quais os parasitas não apresentem resistência.

Segundo o Ministério da Saúde, o uso de antimonial pentavalente deve ser a droga de primeira escolha para o tratamento de leishmaniose. Há relatos de que o Antimoniato de meglumina é capaz de induzir toxicidade renal e cardíaca após sua administração (Oliveira et al., 2017). Também há um relato que uma paciente do sexo feminino, de 58 anos, lavradora, 
procedente da área rural do Município de Cianorte, Paraná evoluiu a óbito, dentre outros fatores, devido aos efeitos tóxicos causados pela administração de antimoniato de N-metil glucamina para o tratamento da leishmaniose (M. V. N. de Lima et al., 2007).

De acordo com alguns relatos as proteínas Tiol-redutase dependente (TDR) SbV-AsV redutase (LmACR2) e a proteína de membrana aquagliceroporina estão relacionadas com o mecanismo de resistência de espécies de Leishmania sp. As proteínas TDR e SbV-AsV estão relacionadas com a conversão do SbV em antimonial trivalente (SbIII), se essa conversão não ocorrer, não haverá o tratamento efetivo da leishmaniose ocasionado por essa droga (Ponte-Sucre et al., 2017). Paralelo a isso, a resistência aos antimoniais também está associado aos baixos níveis de SbIII intracelular nos parasitas. A baixa expressão de aquagliceroporina ocasiona baixos níveis intracelulares de SbIII, assim é possível inferir que essa proteína também possui influencia no mecanismo de resistência do parasita (Moreira et al., 2018).

Para o desenvolvimento de medicamentos contra a leishmaniose novos alvos moleculares do parasita devem ser descobertos. A enzima Diidroorotato desidrogenase (DHODH) tem sido relatada como um promissor alvo para ação de compostos antiparasitários. Pois essa enzima é encontrada em parasitas da família Trypanosomatidae da qual fazem parte o Trypanosoma sp. e Leishmania sp. (Chibli et al., 2018; Reis et al., 2015).

A DHODH participa da quarta etapa da via de síntese de novo de pirimidinas. Essa via de síntese é essencial para esses parasitas, sendo assim, se uma de suas enzimas sofrer inibição o crescimento e/ou sobrevivência do parasita pode ser comprometida (Weetall et al., 2019).

Recentemente estudos de knockout e knockdwn foram realizados para, respectivamente, impedir a expressão do gene de DHODH de Tripanossoma cruzi que levou a inviabilidade celular do parasita (Liu et al., 2017); e para reduzir a expressão do gene DHODH de T. brucei que levou a diminuição do crescimento do parasita (Wu et al., 2018).

Abordagens de modelagem molecular tem sido utilizadas com sucesso para o planejamento de novos compostos (Araújo et al., 2020; da Silva Júnior et al., 2021; Leão et al., 2020; Neto et al., 2020; M. S. de Oliveira et al., 2020). Dessa forma, neste artigo, usamos métodos in silico para propor a estrutura tridimensional da enzima DHODH, além de novos compostos nitroderivados. Esses novos compostos foram construídos utilizando o Metronidazol e Benzonidazol (Figura 1) como arcabouços moleculares. Justifica-se a escolha desses fármacos, devido eles apresentarem atividade antimicrobiana e antiparasitária (Alves et al., 2020; Hof, 1989; Oliveira et al., 2018; Pelozo et al., 2021; Raether \& Hänel, 2003). Além disso, eles possuem como mecanismo reação a oxirredução, mesmo mecanismo encontrado na via de síntese de novo da DHODH. Também avaliamos o modo de interação desses compostos com o local de ligação da proteína.

Figura 1. Estrutura molecular do (A) Metronidazol e (B) Benzonidazol.

(A)

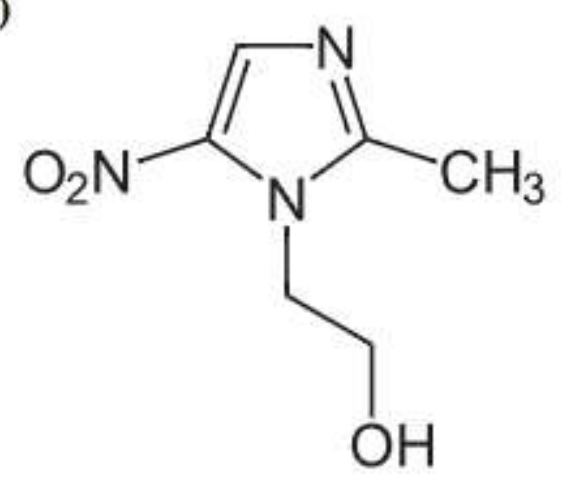

(B)

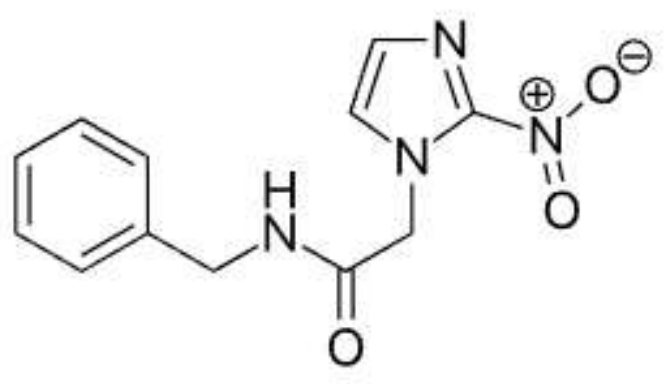

Fonte: Autores. 


\section{Metodologia}

\subsection{Modelagem molecular por homologia}

A construção de proteínas com estrutura tridimensional desconhecida a partir de modelos de homologia é importante ferramenta para o estudo das interações, proposta de novos inibidores e mecanismos catalíticos, na finalidade de promover o estudo de novos fármacos (Neves Cruz et al., 2020; Ramos et al., 2020; Santos et al., 2020). Assim, o primeiro passo deste estudo foi localizar a sequência fasta da DHODH de Leishimania amazonensis. A sequência fasta dessa proteína pode ser acessada no Uniprot a partir do código Q9NL74. A construção da proteína foi baseada na identidade e similaridade entre as enzimas DHODH de Leishmania major (PDB:3TQ0) e Leishimania amazonenses. A estrutura tridimensional da proteína foi construída utilizando o SWISS-MODEL (Costa et al., 2020; Santana de Oliveira et al., 2021).

\subsection{Design dos compostos e docking molecular}

O design dos compostos MTZ1 e MTZ2 foi realizado a partir da combinação de estruturas privilegiadas do Metronidazol e Benzonidazol. Esses estruturas privilegiadas são as principais regiões da molécula que são responsáveis pela sua atividade biológica. A seleção dessas regiões foi realizada a partir de estudos anteriores que relatam a importância desses grupos moleculares para função biológica (Moreno-Herrera et al., 2020; Patel et al., 2021; Ramya, 2016; Yerragunta et al., 2014). Para avaliar a estrutura e reatividade dos derivados foram utilizados cálculos computacionais para avaliar as regiões de HOMO, LUMO e GAP dos compostos (Figura 3).

Figura 3. Estrutura molecular do derivado (A) MTZ 1 e (B) MTZ 2.

(A)

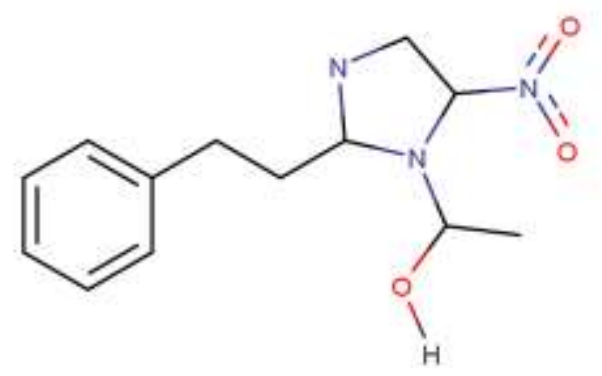

(B)

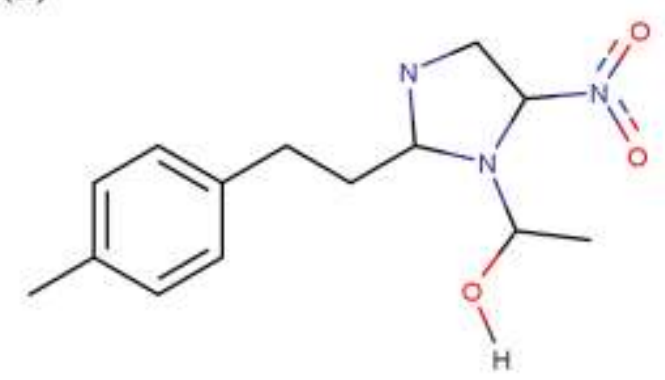

Fonte: Autores.

A partir da análise estrutural e eletrônica é possível determinar as regiões envolvidas em interações eletrostáticas e em mecanismos de transferência de carga entre os compostos estudados e receptores biológicos. Os mapas de HOMO e LUMO indicam as regiões nucleofílicas e eletrofílicas, respectivamente.

Neste trabalho utilizamos o software Molegro Virtual Molegro 5.5 (Almeida et al., 2021; Castro et al., 2021; A. R. J. A. de M. Lima et al., 2020; Thomsen \& Christensen, 2006) para avaliar a interação dos análogos do MTZ e BNZ com o local de ligação da proteína. A função de pontuação MolDock Score (GRID) será utilizada com Grid resolution de $0.30 \AA ̊$ e raio de 7 Å englobando todo o local de ligação. 


\section{Resultados e Discussão}

\subsection{Modelagem molecular por homologia}

Inicialmente foi realizado o alinhamento entre a sequência primária da enzima de Leishmania amazonensis e a sequência primaria da enzima DHODH de Leishmania major (PDB: 3TQ0) publicada no banco de dados de proteínas (PDB) (Cheleski et al., 2010).

A partir dos resultados gerados pelo alinhamento no ALINE, observa-se na Figura 4 que a sequência primária de Leishmania amazonensis apresenta identidade de $83.9 \%$ e similaridade de $86.5 \%$ com a sequência primária de Leishmania major. Isso é corroborado pelo fato de a Dihidroorotato desidrogenase das espécies de Leishmania possuírem seus domínios conservados (Marchler-Bauer et al., 2017). Na literatura encontra-se um valor de identidade sequencial para que possa ser executada a modelagem molecular por homologia, como significante um valor acima de $30 \%$ de identidade sequencial entre a proteína-molde e a proteína-alvo (Xu et al., 2008).

Figura 4. Alinhamento entre as sequências primárias de DHODH de Leishmania amazonensis e Leishmania major (3TQ0) fornecido pelo programa ALINE.

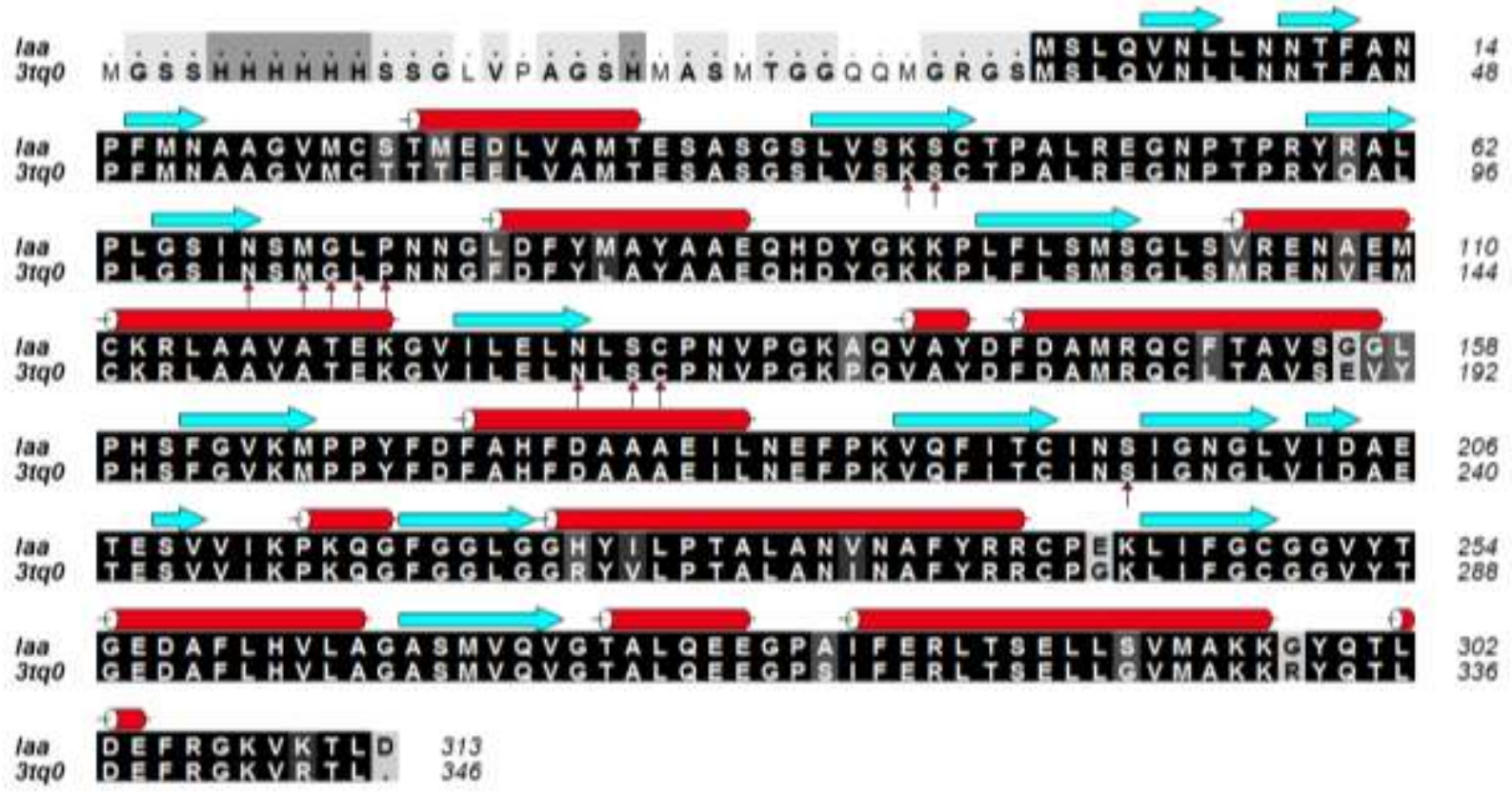

Fonte: Autores.

Se o grau de identidade for igual ou superior a cerca de $25 \%$ entre as estruturas primárias das proteínas-molde e da proteína alvo, com números de resíduos superior a 80, existe grande probabilidade de que estas proteínas tenham estruturas tridimensionais semelhantes, e pode-se construir um modelo para a proteína alvo (Santos Filho \& Alencastro, 2003).

Após alinhamento da estrutura primária de Leishmania amazonensis e Leishmania major foi construído o modelo tridimensional para a enzima DHODH de Leishmania amazonensis, esse modelo foi gerado no programa SWISS-MODEL (Costa et al., 2020; Santana de Oliveira et al., 2021), os loops foram otimizados através das derivadas das restrições estereoquímicas para buscar modelos energeticamente mais estáveis (Figura 5). Além disso, o modelo foi avaliado na intenção de determinar qual o melhor modelo gerado na homologia. 
Figura 5. Modelo tridimensional de DHODH de Leishmania amazonenesis gerado pelo SWISS-MODEL.

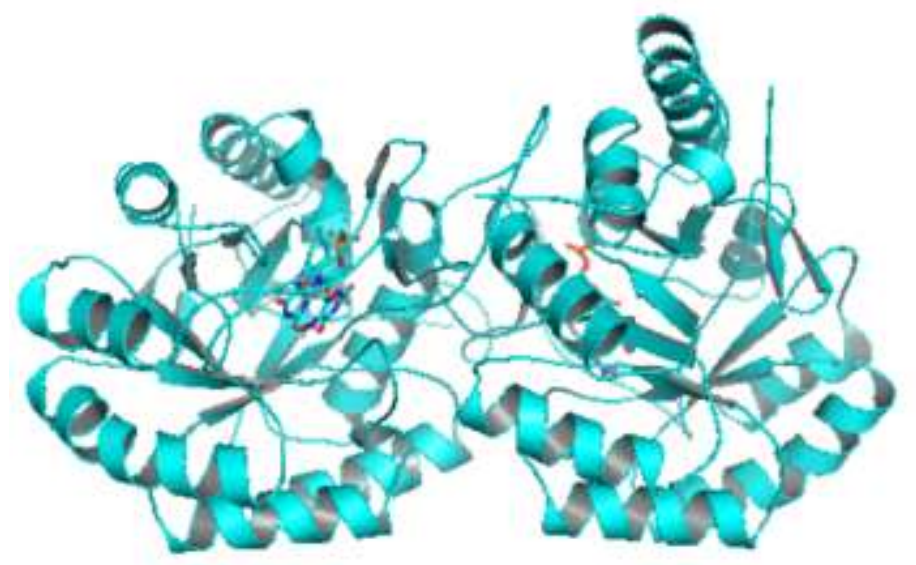

Fonte: Autores.

A Figura 5 apresenta o modelo gerado após a modelagem molecular por homologia, este modelo apresenta 311 resíduos, dispostos entre a Metionina 1 e Leucina 311. A enzima modelada apresenta 9 estruturas secundárias $\alpha$-hélices, 12 estruturas secundárias $\beta$-folhas, completando o modelo regiões de alças interligando as $\alpha$-hélice e as $\beta$-folhas.

A avaliação estereoquímica do modelo foi feita através do gráfico de Ramachandran do servidor RAMPAGE. O número de resíduos localizados em regiões com ângulos favoráveis, dados em porcentagem, para o modelo foi considerado satisfatório, o modelo gerado do domínio catalítico apresentou $96 \%$ dos resíduos previstos dentro da região favorável, $4 \%$ regiões permitidas e 0 resíduos em região de outlier (Figura 6). Esta é uma análise estatística dos de aminoácidos em cada região e permissões, e isto é visualizado também para resíduos de prolina e glicina (triângulo), mesmo que estes apresentem permissões diferentes em relação aos demais aminoácidos, devido as suas flexibilidades (Lovell et al., 2003).

Figura 6. Gráfico de RAMACHANDRAN obtido através do servidor Rampage para validação do modelo 3D da $L a D H O D H$.

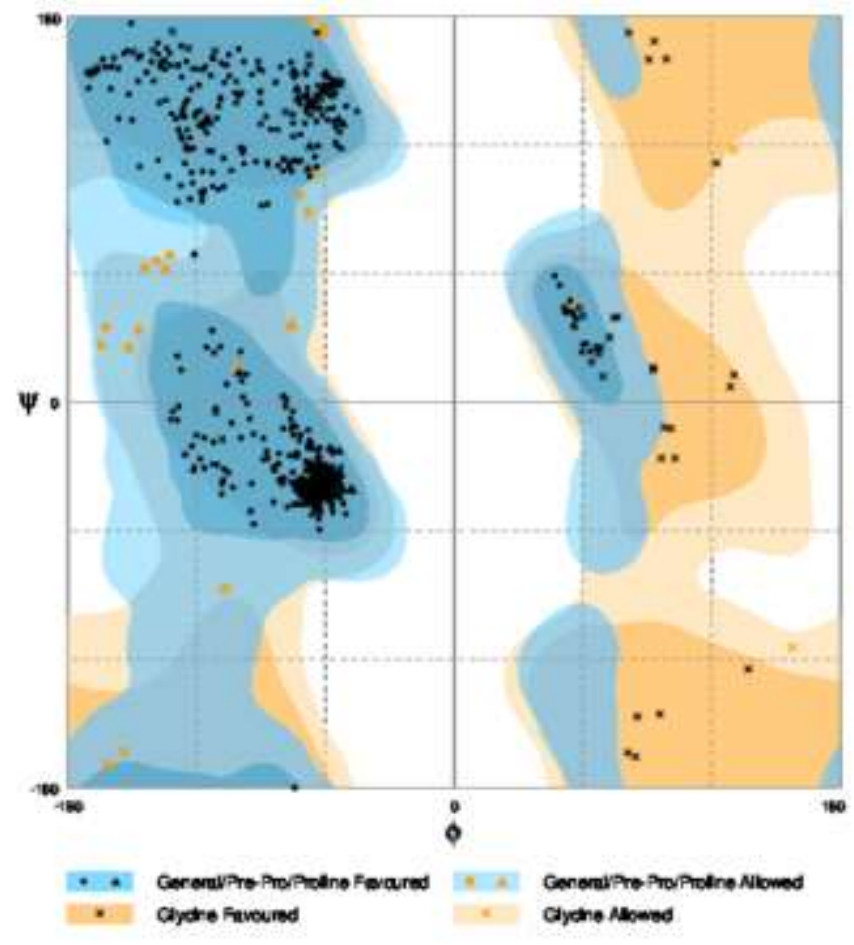

Fonte: Autores. 
O gráfico de ANOLEA (Atomic Non-Local Environment Assessment), também disponível pelo SwissModel, calcula o somatório da interação dos átomos, para cada um dos aminoácidos resultando na obtenção da energia potencial de uma proteína, demonstrando se o ambiente químico ao redor do átomo contribui ou não para sua estabilidade. A partir disso é gerada uma pontuação para cada aminoácido que é analisado graficamente, podendo determinar assim zonas com elevada energia, geralmente relacionadas a erros estereoquímicos e choques ou interações indevidas (Melo \& Feytmans, 1998).

O gráfico de ANOLEA á apresentado na Figura 7 acima é usado para avaliar a qualidade do modelo, e mostra a energia de interação de cada resíduo de uma cadeia de proteína podendo determinar assim zonas com elevada energia, geralmente relacionadas a erros estereoquímicos e choques ou interações indevidas. $\mathrm{O}$ gráfico mostra como resultado que mais de $96 \%$ dos resíduos se apresentam em regiões favoráveis energeticamente. O eixo y representa a energia para cada aminoácido (eixo x) da cadeia de proteína. Valores negativos (em verde) apresentam ambientes energeticamente favoráveis (Melo; Feytmans, 1997).

Figura 7. Gráfico de ANOLEA obtido através do servidor SWISS-MODEL para validação do modelo 3D da enzima DHODH.

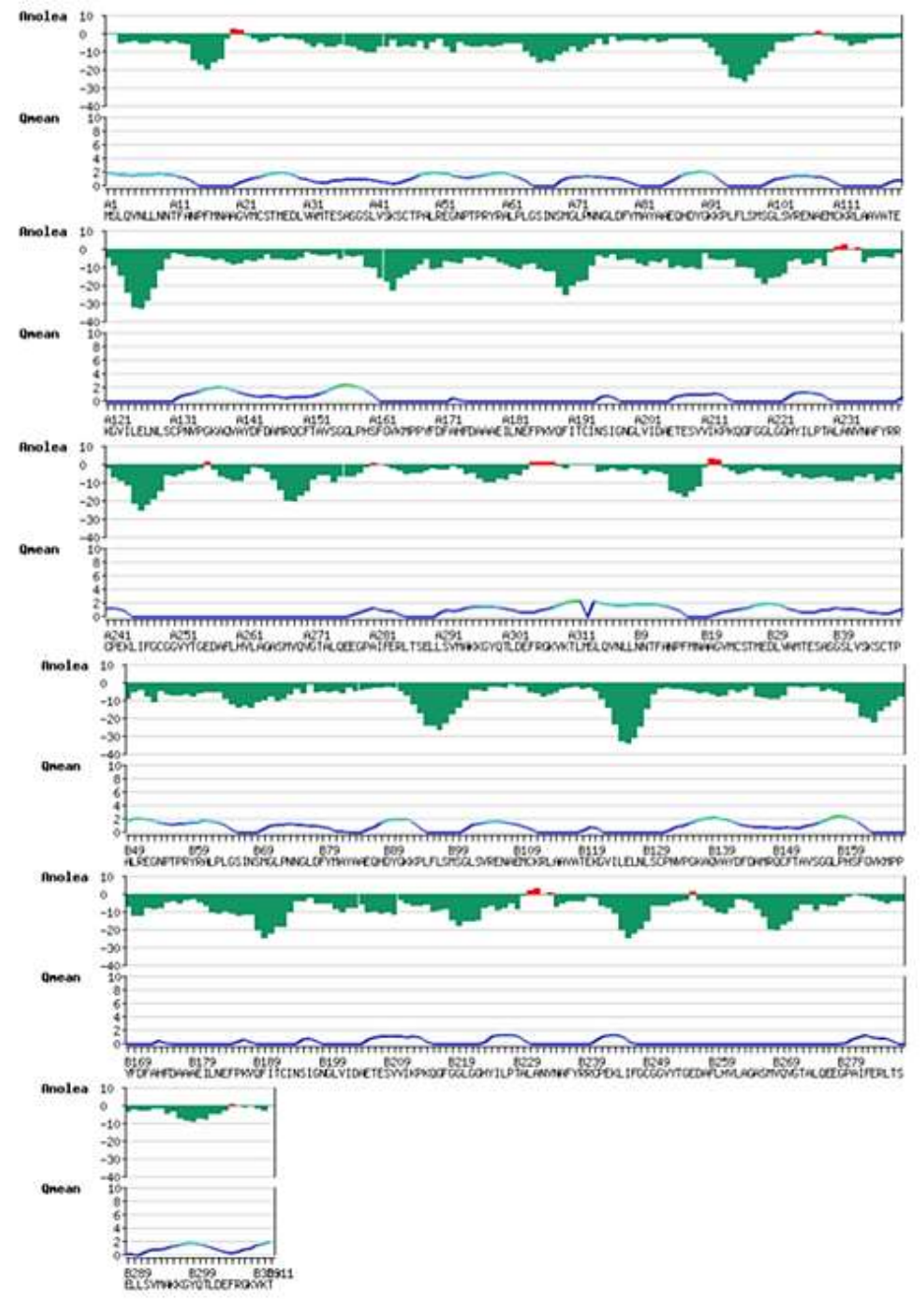

Fonte: Autores.

\subsection{Design dos compostos e docking molecular}

A análise de HOMO e LUMO demonstram as regiões que contribuem para a formação destes orbitais e quanto contribuem para a nucleofilicidade e eletrofilicidade, respectivamente. Portanto, o comportamento redox desta molécula está relacionado a doação ou abstração de elétrons com estas regiões, os quais são responsáveis pela estabilização dos elétrons 
desemparelhados. Os valores de HOMO, LUMO e GAP podem ser observados na Tabela 1. As regiões de HOMO e LUMO podem ser visualizadas na Figura 8.

Tabela 1. Valores dos orbitais de HOMO, LUMO, GAP.

\begin{tabular}{cccc}
\hline Compostos & $\begin{array}{c}\text { HOMO } \\
(\mathrm{eV})\end{array}$ & $\begin{array}{c}\text { LUMO } \\
(\mathrm{eV})\end{array}$ & Gap \\
\hline MTZ1 & -5.98 & -2.47 & 3.51 \\
\hline MTZ2 & -5.85 & -2.41 & 3.44 \\
\hline \multicolumn{5}{c}{ Fonte: Autores. }
\end{tabular}

Figura 8. Análise gráfica dos coeficientes dos orbitais moleculares de HOMO e LUMO dos derivados.

HOMO

MTZ1

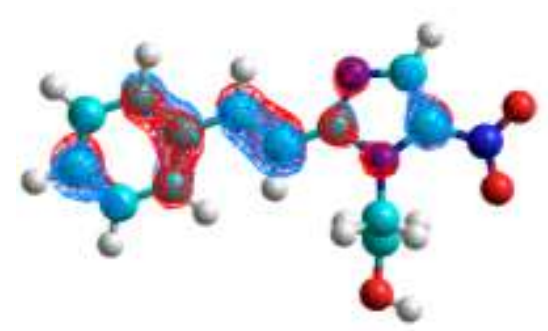

MTZ2

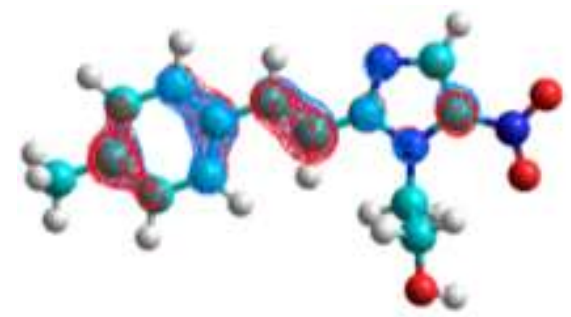

LUMO
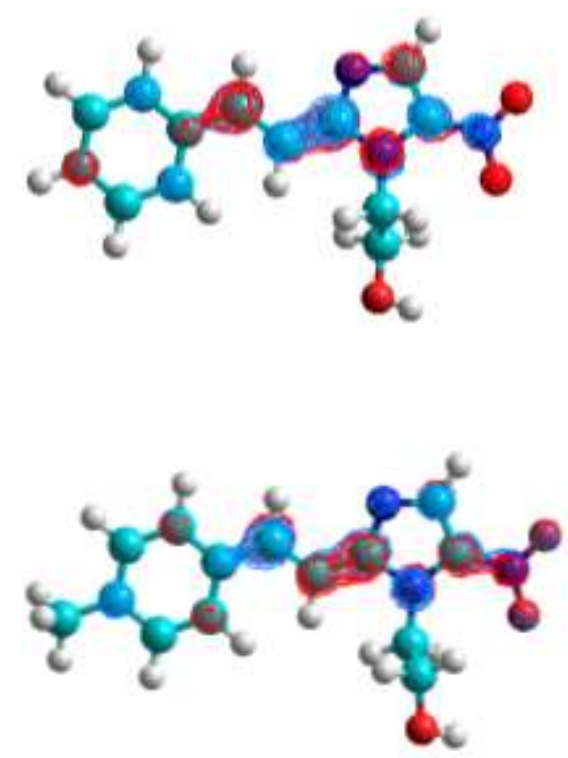

Fonte: Autores.

O acoplamento molecular ou docking proporciona o conhecimento básico das interações ligante-receptor, demonstrando uma estimativa de afinidade para futura otimização do ligante. Estudos anteriores demonstraram a identificação de novas regiões a partir do bolso do sítio ativo da DHODH potencialmente capazes de acomodar ligantes, o que afetariam de forma direta na capacidade catalítica da enzima (Cheleski, 2010). Logo, é possível a acoplar novos ligantes com peso molecular e estruturas diferentes que o substrato. Os resultados obtidos para a energia de afinidade dos ligantes com o sítio receptor da DHODH podem ser visualizados na Tabela 2.

Tabela 2. Parâmetros obtidos no docking para o EJZ, BNZ, MTZ, BNMTZ e derivados.

\begin{tabular}{ll}
\hline Compostos & MolDock Score \\
\hline MTZ1 & -80.7144 \\
MTZ2 & -63.6399 \\
\hline
\end{tabular}

Fonte: Autores. 
Observa-se que para o MTZ1 o orbital LUMO encontra-se na porção nitroimidazólica, e se caracteriza como uma região eletrofílica, com boa capacidade de acepção de elétrons. De fato, sabe-se que a atividade biológica de alguns nitrocompostos está diretamente relacionada com o potencial de redução do grupo nitro (Rozenski, 1995).

Os orbitais de HOMO estão presentes na porção do estireno para os derivado MTZ1, além de mostrar uma discreta distribuição ao longo da estrutura. Esta região está relacionada ao ataque nucleofílico da molécula ou o aumento da afinidade por seus alvos (Grant \& Pickup, 1997). Para os derivados de MTZ1 e 2, observa-se que as substituições no anel aromático foram feitas apenas por doadores de elétrons. De um modo geral os derivados estiril-nitroimidazólicos apresentaram melhores valores de HOMO. Compostos com alta energia de HOMO possuem também alto potencial químico. Neste caso a tendência do composto é a de doar elétrons, mas também pode estar vinculado a maior capacidade de estabilizar o radical nitroxil após a acepção de um elétron (Pfaendner, 2006).

A análise do docking para o derivado MTZ1 nos permite observar a manutenção das ligações: Ligação do nitrogênio

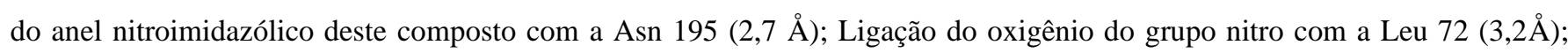
Ligação do nitrogênio doanel nitroimidazólico e do oxigênio da cadeia lateral com o cofator FMN (3,0 e 2,8 $)$, e interações adicionais da hidroxila fenólica com uma Ser 66 (3,0 Å). A análise do docking para o derivado MTZ2 nos permite observar a manutenção das ligações: Ligação do nitrogênio do anel nitroimidazólico deste composto com a Ans 195 (2,8 ̊̊); Ligação do oxigênio do grupo nitro com a Cys 131 (2,5 ̊̊); Ligação do nitrogênio do anel nitroimidazólico e do oxigênio da cadeia lateral com o cofator FMN (3,1 e 3,3 ̊̊). Além de interações adicionais da hidroxila fenólica e metoxila com uma Ser 66 (3,0 ̊).

\section{Conclusão}

Em relação ao modelo construído por homologia molecular, os gráficos demonstraram que mais de $96 \%$ dos resíduos se apresentam em regiões energeticamente favoráveis, demonstrando que a homologia foi satisfatória para a construção da alça no sítio ativo da $L a \mathrm{DHODH}$, demonstrando a validação do método. Os ligantes foram capazes de interagir de maneira favorável com o local de ligação da proteína, conforme observado nos resultados de docking. O derivados MTZ1 e 2 de um modo geral apresentaram valores de HOMO e LUMO que estão intimamente relacionados com sua atividade biológicas. Compostos com alta energia de HOMO possuem também alto potencial químico. Neste caso a tendência do composto é a de doar elétrons, mas também pode estar vinculado a maior capacidade de estabilizar o radical nitroxil após a acepção de um elétron.

\section{Referências}

Akhoundi, M., Kuhls, K., Cannet, A., Votýpka, J., Marty, P., Delaunay, P., \& Sereno, D. (2016). A Historical Overview of the Classification, Evolution, and Dispersion of Leishmania Parasites and Sandflies. PLoS Neglected Tropical Diseases, 10(3), e0004349. https://doi.org/10.1371/journal.pntd.0004349

Almeida, V. M., Dias, Ê. R., Souza, B. C., Cruz, J. N., Santos, C. B. R., Leite, F. H. A., Queiroz, R. F., \& Branco, A. (2021). Methoxylated flavonols from Vellozia dasypus Seub ethyl acetate active myeloperoxidase extract: in vitro and in silico assays . Journal of Biomolecular Structure and Dynamics, 1-10. https://doi.org/10.1080/07391102.2021.1900916

Alves, F. S., Rodrigues Do Rego, J. de A., Da Costa, M. L., Lobato Da Silva, L. F., Da Costa, R. A., Cruz, J. N., \& Brasil, D. D. S. B. (2020). Spectroscopic methods and in silico analyses using density functional theory to characterize and identify piperine alkaloid crystals isolated from pepper (Piper Nigrum L.). Journal of Biomolecular Structure and Dynamics, 38(9), 2792-2799. https://doi.org/10.1080/07391102.2019.1639547

Araújo, P. H. F., Ramos, R. S., da Cruz, J. N., Silva, S. G., Ferreira, E. F. B., de Lima, L. R., Macêdo, W. J. C., Espejo-Román, J. M., Campos, J. M., \& Santos, C. B. R. (2020). Identification of potential COX-2 inhibitors for the treatment of inflammatory diseases using molecular modeling approaches. Molecules, 25(18), 4183. https://doi.org/10.3390/molecules25184183

Arenas, R., Torres-Guerrero, E., Quintanilla-Cedillo, M. R., \& Ruiz-Esmenjaud, J. (2017). Leishmaniasis: A review. In F1000Research (Vol. 6). Faculty of 1000 Ltd. https://doi.org/10.12688/f1000research.11120.1

Castro, A. L. G., Cruz, J. N., Sodré, D. F., Correa-Barbosa, J., Azonsivo, R., de Oliveira, M. S., de Sousa Siqueira, J. E., da Rocha Galucio, N. C., de Oliveira Bahia, M., Burbano, R. M. R., do Rosário Marinho, A. M., Percário, S., Dolabela, M. F., \& Vale, V. V. (2021). Evaluation of the genotoxicity and mutagenicity of isoeleutherin and eleutherin isolated from Eleutherine plicata herb. using bioassays and in silico approaches. Arabian Journal of Chemistry, 
14(4), 103084. https://doi.org/10.1016/j.arabjc.2021.103084

CDC's Division of Parasitic Diseases and Malaria. (2014). Guide for Specimen Collection and Reference Diagnosis of Leishmaniasis (p. 4).

Cheleski, J., Rocha, J. R., Pinheiro, M. P., Wiggers, H. J., Da Silva, A. B. F., Nonato, M. C., \& Montanari, C. A. (2010). Novel insights for dihydroorotate dehydrogenase class 1A inhibitors discovery. European Journal of Medicinal Chemistry, 45(12), 5899-5909. https://doi.org/10.1016/j.ejmech.2010.09.055

Chibli, L. A., Schmidt, T. J., Nonato, M. C., Calil, F. A., \& Da Costa, F. B. (2018). Natural products as inhibitors of Leishmania major dihydroorotate dehydrogenase. European Journal of Medicinal Chemistry, 157, 852-866. https://doi.org/10.1016/j.ejmech.2018.08.033

Costa, E. B. B., Silva, R. C. C., Espejo-Román, J. M. M., Neto, M. F. de A. F. d. A., Cruz, J. N. N., Leite, F. H. A. H. A., Silva, C. H. T. P. H. T. P., Pinheiro, J. C. C., Macêdo, W. J. C. J. C., \& Santos, C. B. R. B. R. (2020). Chemometric methods in antimalarial drug design from 1,2,4,5-tetraoxanes analogues. SAR and QSAR in Environmental Research, 31(9), 1-19. https://doi.org/10.1080/1062936X.2020.1803961

da Silva Júnior, O. S., Franco, C. de J. P., de Moraes, A. A. B., Cruz, J. N., da Costa, K. S., do Nascimento, L. D., \& Andrade, E. H. de A. (2021). In silico analyses of toxicity of the major constituents of essential oils from two Ipomoea L. species. Toxicon, 195, 111-118. https://doi.org/10.1016/j.toxicon.2021.02.015

Grant, J. A., \& Pickup, B. T. (1997). Gaussian shape methods. Computer Simulation of Biomolecular Systems, 150-176. https://doi.org/10.1007/978-94-0171120-3_5

Hof, H. (1989). Antibacterial activities of the antiparasitic drugs nifurtimox and benznidazole. Antimicrobial Agents and Chemotherapy, 33(3), 404-405. https://doi.org/10.1128/AAC.33.3.404

Leão, R. P., Cruz, J. V. J. N., da Costa, G. V., Cruz, J. V. J. N., Ferreira, E. F. B., Silva, R. C., de Lima, L. R., Borges, R. S., Dos Santos, G. B., \& Santos, C. B. R. (2020). Identification of new rofecoxib-based cyclooxygenase-2 inhibitors: A bioinformatics approach. Pharmaceuticals, 13(9), 1-26. https://doi.org/10.3390/ph13090209

Lima, A. R. J. A. de M., Siqueira, A. S., Möller, M. L. S., Souza, R. C. de, Cruz, J. N., Lima, A. R. J. A. de M., Silva, R. C. da, Aguiar, D. C. F., Junior, J. L. da S. G. V., \& Gonçalves, E. C. (2020). In silico improvement of the cyanobacterial lectin microvirin and mannose interaction. Journal of Biomolecular Structure and Dynamics. https://doi.org/10.1080/07391102.2020.1821782

Lima, M. V. N. de, Oliveira, R. Z. de, Lima, A. P. de, Cerino, D. A., \& Silveira, T. G. V. (2007). Leishmaniose cutânea com desfecho fatal durante tratamento com antimonial pentavalente. Anais Brasileiros de Dermatologia, 82, 269-271. http://www.scielo.br/scielo.php?script=sci_arttext\&pid=S036505962007000300010\&nrm=iso

Limeira, C. H., Alves, C. J., De Azevedo, S. S., Santos, C. D. S. A. B., De Melo, M. A., Soares, R. R., Barnabé, N. N. D. C., \& Rodrigues, G. D. Q. (2019). Clinical aspects and diagnosis of leishmaniasis in equids: A systematic review and meta-analysis. Revista Brasileira de Parasitologia Veterinaria, 28(4), 574581. https://doi.org/10.1590/s1984-29612019074

Liu, L., Dong, Z., Lei, Q., Yang, J., Hu, H., Li, Q., Ji, Y., Guo, L., Zhang, Y., Liu, Y., \& Cui, H. (2017). Inactivation/deficiency of DHODH induces cell cycle arrest and programed cell death in melanoma. Oncotarget, 8(68), 112354-112370. https://doi.org/10.18632/oncotarget.19379

Lovell, S. C., Davis, I. W., Arendall, W. B., De Bakker, P. I. W., Word, J. M., Prisant, M. G., Richardson, J. S., \& Richardson, D. C. (2003). Structure validation by Calpha geometry: phi,psi and Cbeta deviation. Proteins, 50(3), 437-450. https://doi.org/10.1002/PROT.10286

Mandlik, V., \& Singh, S. (2016). Integrative approaches for identification of novel ISCL inhibitors in Leishmaniasis: A computational insight into the structure. Gene Reports, 4, 162-168. https://doi.org/10.1016/j.genrep.2016.05.002

Marchler-Bauer, A., Bo, Y., Han, L., He, J., Lanczycki, C. J., Lu, S., Chitsaz, F., Derbyshire, M. K., Geer, R. C., Gonzales, N. R., Gwadz, M., Hurwitz, D. I., Lu, F., Marchler, G. H., Song, J. S., Thanki, N., Wang, Z., Yamashita, R. A., Zhang, D., ... Bryant, S. H. (2017). CDD/SPARCLE: Functional classification of proteins via subfamily domain architectures. Nucleic Acids Research, 45(D1), D200-D203. https://doi.org/10.1093/nar/gkw1129

Melo, F., \& Feytmans, E. (1998). Assessing protein structures with a non-local atomic interaction energy. Journal of Molecular Biology, 277(5), 1141-1152. https://doi.org/10.1006/jmbi.1998.1665

Ministério da Saúde. (2017). Manual de Vigilância da Leishmaniose Tegumentar Americana. In Ministério da Saúde.Secretaria de Vigilância em Saúde.

Moreira, D. de S., Xavier, M. V., \& Murta, S. M. F. (2018). Ascorbate peroxidase overexpression protects leishmania Braziliensis against trivalent antimony effects. Memorias Do Instituto Oswaldo Cruz, 113(12), 1-5. https://doi.org/10.1590/0074-02760180377

Moreno-Herrera, A., Cortez-Maya, S., Bocanegra-Garcia, V., Banik, B. K., \& Rivera, G. (2020). Recent Advances in the Development of Broad-Spectrum Antiprotozoal Agents. Current Medicinal Chemistry, 28(3), 583-606. https://doi.org/10.2174/09298667327666200303170000

Neto, R. de A. M. M., Santos, C. B. R. R., Henriques, S. V. C. C., Machado, L. de O., Cruz, J. N., da Silva, C. H. T. d. P. T. de P., Federico, L. B., Oliveira, E. H. C. d. C. de, de Souza, M. P. C. C., da Silva, P. N. B. B., Taft, C. A., Ferreira, I. M., \& Gomes, M. R. F. F. (2020). Novel chalcones derivatives with potential antineoplastic activity investigated by docking and molecular dynamics simulations. Journal of Biomolecular Structure and Dynamics, 1-13. https://doi.org/10.1080/07391102.2020.1839562

Neves Cruz, J., da Costa, K. S., de Carvalho, T. A. A., \& de Alencar, N. A. N. (2020). Measuring the structural impact of mutations on cytochrome P450 21A2, the major steroid 21-hydroxylase related to congenital adrenal hyperplasia. Journal of Biomolecular Structure and Dynamics, 38(5), 1425-1434. https://doi.org/10.1080/07391102.2019.1607560

No, J. H. (2016). Visceral leishmaniasis: Revisiting current treatments and approaches for future discoveries. Acta Tropica, 155, 113-123. https://doi.org/10.1016/j.actatropica.2015.12.016

Oliveira, A. A., Oliveira, A. P. A., Franco, L. L., Ferencs, M. O., Ferreira, J. F. G., Bachi, S. M. P. S., Speziali, N. L., Farias, L. M., Magalhães, P. P., \& 
Beraldo, H. (2018). 5-Nitroimidazole-derived Schiff bases and their copper(II) complexes exhibit potent antimicrobial activity against pathogenic anaerobic bacteria. BioMetals 2018 31:4, 31(4), 571-584. https://doi.org/10.1007/S10534-018-0106-6

Oliveira, M. S. de, Cruz, J. N. da, Costa, W. A. da, Silva, S. G., Brito, M. da P., Menezes, S. A. F. de, Neto, A. M. de J. C., Andrade, E. H. de A., \& Junior, R. N. de C. (2020). Chemical Composition, Antimicrobial Properties of Siparuna guianensis Essential Oil and a Molecular Docking and Dynamics Molecular Study of its Major Chemical Constituent. Molecules, 25(17), 3852. https://doi.org/10.3390/molecules25173852

Oliveira, L. F. G., Souza-Silva, F., Cysne-Finkelstein, L., Rabelo, K., Amorim, J. F., Azevedo, A. de S., Bourguignon, S. C., Ferreira, V. F., Paes, M. V., \& Alves, C. R. (2017). Evidence for Tissue Toxicity in BALB/c Exposed to a Long-Term Treatment with Oxiranes Compared to Meglumine Antimoniate. BioMed Research International, 2017, 9840210. https://doi.org/10.1155/2017/9840210

Patel, O. P. S., Jesumoroti, O. J., Legoabe, L. J., \& Beteck, R. M. (2021). Metronidazole-conjugates: A comprehensive review of recent developments towards synthesis and medicinal perspective. European Journal of Medicinal Chemistry, 210, 112994. https://doi.org/10.1016/J.EJMECH.2020.112994

Pelozo, M. F., Lima, G. F. S., Cordeiro, C. F., Silva, L. S., Caldas, I. S., Carvalho, D. T., Lavorato, S. N., Hawkes, J. A., \& Franco, L. L. (2021). Synthesis of New Hybrid Derivatives from Metronidazole and Eugenol Analogues as Trypanocidal Agents. Journal of Pharmacy \& Pharmaceutical Sciences, 24, 421-434. https://doi.org/10.18433/JPPS31839

Pfaendner, R. (2006). Nitroxyl radicals and nitroxylethers beyond stabilization: radical generators for efficient polymer modification. Comptes Rendus Chimie, 9(11-12), 1338-1344. https://doi.org/10.1016/J.CRCI.2006.08.001

Ponte-Sucre, A., Gamarro, F., Dujardin, J. C., Barrett, M. P., López-Vélez, R., García-Hernández, R., Pountain, A. W., Mwenechanya, R., \& Papadopoulou, B. (2017). Drug resistance and treatment failure in leishmaniasis: A 21st century challenge. In PLoS Neglected Tropical Diseases (Vol. 11, Issue 12, p. e0006052). Public Library of Science. https://doi.org/10.1371/journal.pntd.0006052

Raether, W., \& Hänel, H. (2003). Nitroheterocyclic drugs with broad spectrum activity. Parasitology Research 2003 90:1, 90(1), S19-S39. https://doi.org/10.1007/S00436-002-0754-9

Ramos, R. S., Macêdo, W. J. C., Costa, J. S., da Silva, C. H. T. d. P., Rosa, J. M. C., da Cruz, J. N., de Oliveira, M. S., de Aguiar Andrade, E. H., e Silva, R. B. L., Souto, R. N. P., \& Santos, C. B. R. (2020). Potential inhibitors of the enzyme acetylcholinesterase and juvenile hormone with insecticidal activity: study of the binding mode via docking and molecular dynamics simulations. Journal of Biomolecular Structure and Dynamics, 38(16), 4687-4709. https://doi.org/10.1080/07391102.2019.1688192

Ramya, N. (2016). Design, Synthesis, Characterization and Biological Evaluation of Some Novel Anti Tubercular Agents Targeting: DecaprenylphosphorylBeta-D-Ribose 2'Epimerase-1.

Reis, R. A. G., Lorenzato, E., Silva, V. C., \& Nonato, M. C. (2015). Recombinant production, crystallization and crystal structure determination of dihydroorotate dehydrogenase from Leishmania (Viannia) braziliensis. Acta Crystallographica Section F:Structural Biology Communications, 71 (Pt 5), 547552. https://doi.org/10.1107/S2053230X15000886

Santana de Oliveira, M., Pereira da Silva, V. M., Cantão Freitas, L., Gomes Silva, S., Nevez Cruz, J., \& de Aguiar Andrade, E. H. (2021). Extraction Yield, Chemical Composition, Preliminary Toxicity of Bignonia nocturna (Bignoniaceae) Essential Oil and in Silico Evaluation of the Interaction. Chemistry and Biodiversity, 18(4), cbdv.202000982. https://doi.org/10.1002/cbdv.202000982

Santos, C. B. R., Santos, K. L. B., Cruz, J. N., Leite, F. H. A., Borges, R. S., Taft, C. A., Campos, J. M., \& Silva, C. H. T. P. (2020). Molecular modeling approaches of selective adenosine receptor type 2A agonists as potential anti-inflammatory drugs. Journal of Biomolecular Structure and Dynamics. https://doi.org/10.1080/07391102.2020.1761878

Santos Filho, O. A., \& Alencastro, R. B. de. (2003). Modelagem de proteínas por homologia. Química Nova, 26(2), 253-259. https://doi.org/10.1590/s010040422003000200019

Thakur, S., Joshi, J., \& Kaur, S. (2020). Leishmaniasis diagnosis: an update on the use of parasitological, immunological and molecular methods. In Journal of Parasitic Diseases 44(2), 253-272. Springer. https://doi.org/10.1007/s12639-020-01212-w

Thomsen, R., \& Christensen, M. H. (2006). MolDock: A new technique for high-accuracy molecular docking. Journal of Medicinal Chemistry, 49(11), 33153321. https://doi.org/10.1021/jm051197e

Weetall, M., Kojima, K., Piya, S., Trotta, C., Baird, J., O’Keefe, K., Furia, B., Borthakur, G. M., \& Spiegel, R. (2019). Inhibition of De Novo Pyrimidine Nucleotide Synthesis By the Novel DHODH Inhibitor PTC299 Induces Differentiation and/or Death of AML Cells. Blood, 134(Supplement_1), 5152-5152. https://doi.org/10.1182/blood-2019-124569

WHO. (2020). Leishmaniasis. https://www.who.int/news-room/fact-sheets/detail/leishmaniasis

Wu, D., Wang, W., Chen, W., Lian, F., Lang, L., Huang, Y., Xu, Y., Zhang, N., Chen, Y., Liu, M., Nussinov, R., Cheng, F., Lu, W., \& Huang, J. (2018). Pharmacological inhibition of dihydroorotate dehydrogenase induces apoptosis and differentiation in acute myeloid leukemia cells. Haematologica, 103(9), 1472-1483. https://doi.org/10.3324/haematol.2018.188185

Xu, Y., Colletier, J. P., Weik, M., Jiang, H., Moult, J., Silman, I., \& Sussman, J. L. (2008). Flexibility of aromatic residues in the active-site gorge of acetylcholinesterase: X-ray versus molecular dynamics. Biophysical Journal, 95(5), 2500-2511. https://doi.org/10.1529/biophysj.108.129601

Yerragunta, V., Gayathri, R., Srujana, S., patil, P., Srujana, S., Devi, R., \& Divya, A. (2014). Benzimidazole Derivatives and its Biological Importance: A Review. PharmaTutor, 2(3), 109-113. http://www.pharmatutorjournal.com/index.php/pt/article/view/66 